\title{
Game-Playing Culture in an Age of Capitalist Consumption: Young Taiwanese and Collectible Card Games
}

\author{
${ }^{1}$ LavanchaweeSujarittanonta, ${ }^{2} \mathrm{John}$ Walsh* \\ 1I-Hsou University, Taiwan \\ ${ }^{2}$ School of Management, Shinawatra University, Thailand \\ *1jcwalsh100@hotmail.com
}

\begin{abstract}
Games-playing has historically been a form of leisure that is social and interactive in nature. As such, notwithstanding the dangers associated with gambling which may also be linked to games-playing, games have been considered a generally worthwhile activity which may have positive behavioral and educational externalities. However, in the age of advanced capitalism, games-playing has become subject to intensive marketing and advertising to promote consumption, particularly among young people who may have the time to play and some disposable income to invest in new games. One question this raises is the extent to which traditional games-playing modes and styles been affected by such marketing. This paper reports on research with a sample of Taiwanese undergraduate students investigating their consumption of games, the extent to which they use games to interact with other people and the changes of behavior with respect to games culture in recent years. A particular focus on the collectible card games segment of the gaming industry lends some focus to the findings described.
\end{abstract}

\section{Keywords: Culture, capitalist consumption, Young Taiwanese}

\section{Introduction}

Games are a form of competitive activity which have specific rules of play and which are conducted for pleasure, either wholly or mostly. According to Sale and Zimmerman (2003:96): "A game is a system in which players engage in an artificial conflict, defined by rules, that results in a quantifiable outcome." There is a very wide range of games, from the traditional forms that have been used in East Asia for centuries to the very modern and innovative online computer games that have become so popular that new releases of leading brands have become globally significant media events. New production methods have made it much more possible for creativity to be converted into marketable products. This has contributed to the plethora of new games, many of which have had a very short life cycle. Others appear to be more durable in appeal. Irrespective of the intentions of the authors and creators involved, new products must jostle for attention in an increasingly crowded marketplace in which some, if not all, competitors are backed by significant marketing support and commercial resources. A subset of games overall that has become extremely popular in recent decades is trading card games (TCG). Derived from the earlier concept of baseball cards, TCGs are played with individually constructed card decks drawn from semi-randomized starter packs and booster packs. Starter packs generally contain sufficient cards for players to start to play the game but a great deal of the entertainment associated with the activity is connected with collecting new cards and planning and deploying various combinations with a view to more successful play. Although themes and styles of play within the TCG category vary, most feature a means of eliminating the other player or players as part of the path towards victory. There is, therefore, a strong incentive for players who enjoy the game to continue to refresh their collections on a regular basis, which of course represents the basic profit opportunity. Players can collect cards by purchasing additional booster packs, swapping or trading with each other or buying desired cards on informal or semi-formal secondary markets organized by players, retailers or dealers.

TCGs are generally high-context in concept and tend to feature fantasy or science fiction themes. This gives the players opportunities to role-play exciting personas or interact with cultural icons. This is especially true in the case of TCGs themed on licensed intellectual property, including such well-known names as Star Trek, Star Wars and Harry Potter. Game play can be complex and many aspects of the ways in which cards are laid out, activated or interact with each other have been patented, which indicates both that the market is competitive and that TCGs rely on a core set of assumptions about the game world involved and the nature of play. The rules of play, although designed to be easy to understand such that players can begin to play as soon as possible, can be required to resolve complex and ambiguous 
situations as a result of the interactions between the numerous new cards introduced by manufacturers to keep the momentum of the brand alive. In very long-lived games, it has become necessary to request players to ban some cards which, through unintended secondary effects, have become 'broken' - that is, they can be used to circumvent the intent of the rules in such a way that game play is seriously degraded. Such cards may be banned by regulation when organized tournament play is involved. Under these circumstances, therefore, it is possible that individual game episodes may be unsatisfying because there is generally an element of the luck of the draw involved in the game and one player may have an initial overwhelming advantage that makes victory inevitable. Alternatively, the interaction between the decks used by players in some cases may be characterized by the inability to affect each other's resources and the game can become protracted and dull in nature. This possibility means that players invest more time and emotional effort into designing and collecting different types of deck. It is evident, in other words, the TCGs represent a form of leisure activity that is strongly embedded in not just the capitalist mode of production, with its constant creative destruction, but also with a form of leisure that contains within itself elements of capitalist accumulation: change and conflict are to some extent the very purpose of the game. In this paper, an exploratory investigation into the nature of TCG playing in Taiwan is attempted and its results set in the context of the developing game market regionally. It continues with a description of the principal brands involved, the culture of game-playing in Taiwan and the extent to which this has been changed by the new games-playing concepts.

\section{Leading Brands}

Magic: the Gathering: Magic: the Gathering, generally known by fans as $M: t G$, was the first successful TCG and was produced by Wizards of the Coast (WOTC), a previously little known company which subsequently became one of the major players in the games industry owing to the success of M:tG. The game established many of the features that have become more or less standard for TCGs, including the concept of players dueling with each other, a technique for designating which cards are active and which are temporarily unavailable ('tapping'), the requirement to draw and deploy certain combinations of cards so as to play other cards and so forth. M:tG became popular principally among young men, of high school or college age, who tended to conform to what is now known as 'geek culture' (McArthur, 2009). Some groups of players can be quite competitive and one of the more notable forms of support for the game has been the organization and sponsorship of a professional tournament for players to join. The 2012 World Player of the Year Tournament offered a first prize of US $\$ 40,000$ to a winner who would emerge victorious from the field of 16 men ranked as the best players in the world (Davis, 2012). Tournament play of this sort is reminiscent of the way in which the Korean government has supported the online computer games industry, as part of its efforts to build and sustain a knowledge-based industry (Walsh and Techavimol, 2009). WOTC has also been energetic in providing support for the games-playing community, with dedicated online space for two-way interactions, video games and merchandising based on the imaginary world in which the game ostensibly takes place and enlisting support from retailers to provide clubs and venues for local tournament or friendly play, as well as taking a role in the secondary markets (and effectively legitimizing this as a result). By contrast with, for example, music or software production companies, TCG producers have encouraged the recycling of their products and have even collaborated with this process by producing very rare cards and special collectors' items in the knowledge that they may subsequently change hands for hundreds of dollars or more. M:tG is an unusual brand in the market not just because it was an innovator but because it has retained its market share while competitors have come and gone. There are now larger brands in the overall TCG market but, as the next sections indicate, they have managed this primarily through growing the market by making games more appealing to different segments previously unexplored.

Pokémon: The launching of the Pokémon TCG signaled the attempt to broaden the appeal of the game concept to a younger age group. This was achieved through the use of the existing Pokémon ('pocket monster') intellectual property and by producing a simpler and streamlined game-playing experience more suitable for younger players. The company involved, Nintendo, provided a full range of marketing support for the game, including video and other media content and a variety of merchandising options. Owing to the different platforms involved, marketing was cross-fertilizing in nature. Customers involved with other aspects of the Pokémon brand were directed towards the TCG from other manifestations of the brand and vice versa. The Pokémon TCG also represented a deliberate attempt to localize the TCG concept to the East Asia market, particularly of course Japan. This has involved switching the artistic style to the anime-manga comic styles more popular there and adding culturally relevant elements to the game play 
experience. Pokémon has been followed by a number of different games drawing upon the cultural and historical heritage of the region. This has had the effect of broadening the potential market further.

Yu-Gi-Oh!: The Yu-Gi-Oh! TCG phenomenon began in 1999 when it took advantage of existing manga comic book intellectual property and it has subsequently become the global leader in the industry, with more than 22 billion cards sold within a decade (David-Marshall, van Dreunen and Wang, 2009). Yu-Gi$\mathrm{Oh}$ ! was originally a serialized comic book aimed at children and has been accompanied by full length animated films and merchandising. Brand owner Konami Digital Entertainment, Inc., has created a wholly-owned subsidiary, 4K Media, located in New York, as a means of marketing the brand outside Asia and this company offers various licensing opportunities for third parties (4K Media, 2013). The scale of the Yu-Gi-Oh! operation indicates the importance of this sector of the games industry and the belief that it can be sustainable for the foreseeable future. As an age-dependent game concept, the company faces the challenge of retaining the interest of players over the course of years and then funneling them towards other, more complex or sophisticated brands that might be more attractive to a more developed personality.

The TCG Market: Annual sales in the TCG industry exceed US\$2.1 billion annually (David-Marshall et al., 2009), with the largest markets being Japan, the US and then Europe. Most of the players are in the child (6-15) or young person (16-24) age ranges, with most M:tG players being in the latter category and Pokémon and Yu-gi-Oh! players in the former. There is a clear gender distinction among players, which increases further with age, since boys significantly outnumber girls in the pastime. Distribution of TCG products takes place through mostly small-scale games specialist retailers or through mass market claims in the case of the brands aimed at younger people. It is also possible to order cards by mail order from various online distributors and specialists, particularly for those involved in the secondary market of trading and exchanging specific cards. Although the TCG sector has indicated the ways in which the games industry has developed into a significant industry in its own right, many of the people involved in it and who support it still retain some sympathy with the smaller-scale specialist games sector of the past. This included often intense emotional investment in the game and, by extension, the company that provides it and the individual writers and designers involved. Most of the games producers were also strongly invested in the games industry and were willing to work for little or no pay to obtain the game play experience desired.

The relationship between customer and company was often intense and any suggestion that the latter was behaving in an overly commercial manner could endanger the relationship. Companies could be excoriated if they did this because of the apparent lack of authenticity. The importance of the imagined world retaining its consistency extends well beyond the willing suspension of disbelief (Martin, 2004). There is, in other words, something of a contradiction between the capitalist nature of the contemporary games industry and the experience of game-play that a large number of participants actually want to experience. As most companies remain small and privately-owned, this has made obtaining accurate data more difficult. Since the number of gamers involved was comparatively small, especially when compared to the profusion and variety of games involved, companies tended not to compete with each other very strongly but informally cooperated to encourage the hobby as a whole to grow. Games production in this sector has been revolutionized first by the possibilities of desktop publishing and second by the ability to outsource printing to low-cost facilities in, for example, India and China. As previously indicated, the success of the large TCG companies means that these norms have now been outgrown (Winkler, 2006).

\section{Marketing Strategies}

TCG companies display in their marketing strategies some of the features of the small-scale games industry to which they once belonged. This is most clearly identifiable in the strength of the relationship between customers and games companies and this is most commonly manifested in the community support programs that are offered. Taking the form of dedicated online space, with the possibility of interacting with the creative talent involved with the brand, these communities are important in demonstrating the authenticity of the company with respect to dedication to the brand, its intellectual property and history and the consistency of game play. This is supplemented by support for tournament play, loyalty schemes, production of special and unique cards for members and participants of certain events and so forth. The essence of the TCG experience is the ability to refresh the game by adding new cards to existing decks or by creating entirely new ones. This has been most commonly approached by creating new sets of booster packs, on a thematic basis, together with any amendments to the rules that 
might be necessitated by new forms of interaction and types of card. However, there has been customer resistance to this approach, particularly among ex-players of a particular game or if it is felt that a new entrant in the market is providing new cards in too aggressive a manner. As a result, there have been several variants introduced, with companies labeling their games as collectible card games (CCGs), living card games (LCGs) and so forth.

These variants aims to limit the power of people willing to invest more money to obtain dominant decks against which normal players can have little or no hope of winning. It is also noticeable that many tournaments, particularly for $\mathrm{M}: \mathrm{tG}$, feature some events in which players are presented with a couple of sealed packs from which to construct a deck rather than use their own intensively-constructed decks. The newer forms of TCGs are also more suitable for players who are resistant to tournament style play, which features competitiveness and some measure of interpersonal aggression at its heart. The larger companies have also been able to cross-fertilize the brand by using different platforms to promote the intellectual property as a whole and in its various manifestations. This has involved video and film, radio, music and podcasts and the full range of communication media. Customer loyalty to a specific brand is also emphasized, which did not occur in the games industry when it was in its early stage. Viral marketing techniques have also been employed - insofar as it is ever possible successfully to marshal viral techniques - on the basis that network externalities exist in the sector and that the more players there are who play the game, the better, more varied and more sustainable will the overall game play experience be.

\section{Business Strategies}

Companies have also employed different forms of business strategy to increase the value of their brands. This has included the often quite fierce protection of patentable aspects of the game, as well as the intellectual property involved. Additionally, companies have employed both vertical and horizontal integration as a means of reaching customers more effectively and controlling the quality of production and distribution, as well as a means of financial management Digital integration of different forms of intellectual property has also proved to be an important means of maximizing exposure to customers in a comparatively short time frame. This has been particularly important when licensed intellectual property is involved, since such contracts are expensive and, when synchronized with the release of feature films in particular, required to return a profit in quite a limited timeframe. To take advantage of the interest created by such properties, TCG companies need to create games that can appeal to a general public which is not necessarily willing to be a committed part of the gaming fraternity. In these cases, games tend to be very simple in nature, especially when compared with TCGs as a whole and, consequently, tend not to offer sustainable game play value. They are often treated, by regular TCG players, as items to be collected rather than games to play. This is different in the case of intellectual property created by the TCG company or otherwise under its control, since - at least when sensitively managed - it can be recreated or reinvented for sustainable competitive advantage. Finally, there have been efforts by TCG companies to internationalize their products, both in terms of game play concepts and the intellectual property concerned and, also, to localize already internationalized concepts to suit better specific local conditions. This includes the use of locally-specific cultural and historical heritage and, also, variations to game play to reflect local practices with respect to other games with which people may be more familiar. How this takes place in the case of Taiwan will be the subject of the next section.

Game Playing in Taiwan: The majority of the Taiwanese people is ethnically Chinese and seems to follow patterns of behavior towards gaming, which historically has been closely associated with gambling, that are characteristic of all ethnic Chinese communities (Loo, Raylu and Oei, 2008). Some evidence suggests that there are specific cultural, social and historical factors which make gambling and problem gambling more prevalent in some societies rather than others (Raylu and Oei, 2004). Social gaming as an activity, therefore, has become embedded in Taiwanese society for a significant period of time. Games played in this context include those involving playing cards of different sorts and Mahjong, as well as casino-based games. These are games that stimulate an intense excitement among the players to a significant extent only when the gambling element is involved; that is, the games themselves are generally very simple, perhaps even trivial in nature and are strictly repetitive. This of course is essential for a successful gambling game, since the calculation of odds is a very important element to all stakeholders. The enjoyment involved in such games are almost wholly external to the game play experience in itself and the games and the equipment necessary to play them are of very low cost (some Mahjong sets 
excepted) since entry barriers to gambling would be counter-productive. These are activities that date to pre-capitalist times. By contrast, TCGs are representatives of a distinctly capitalist age of consumption.

Taiwanese society has been afflicted in recent years both by the impact of the international environment and by the fruits of the success of its own process of rapid industrial development. International economic crises have drained consumer confidence in all sectors. Meanwhile, young people have faced a period of intensifying competitiveness in the educational and labor markets of the island. The rise of China as a regional and even global superpower has further restricted the ability of Taiwanese to express themselves on the international scene. This is certainly a feature of the mindset of young Taiwanese at the present time and it has most commonly led to a hardening of interests towards leisure activities, which has been encouraged both by personal preference and by external pressures. Although there will always be a market for leisure activities, there seems to be little scope for its growth and development at the present time. This does mean that fewer people are keeping up with the changes in the TCGs and other games they play and, also, that fewer people are being drawn into the pastime because of the perception that has been spread of the accumulations and expenditure required to participate fully.

Games Playing in a Time of Capitalist Consumption: TCGs represent a form of game that has made the transition from pre-capitalist to capitalist activity. Game playing in Taiwan has been a symbol of the precapitalist social activities that have brought people together throughout history. Those games were apart from those who succumb to problem gambling - more important as opportunities for people to recreate themselves by personal interaction and to test their status and standing in the universe through investigating whether their luck will bring them winnings. The games themselves are essentially trivial i.e., the odds of winning or losing can be quite easily calculated, despite the luck element, so there is little surprise or strategy inherent in the game beyond basic analysis. They are also cheap to buy or acquire and the enjoyment that they have provided is external to the game play and pieces themselves. This is clearly different in the case of TCGs, since much of the pleasure involved in playing is bound up with ownership of new and different and hard-to-obtain items. Planning expenditure on cards is an inherent part of the enjoyment offered and, particularly in the case of the rarer and more powerful items, the cards themselves have become items of value and represent capital in circulation. They can also be the subject of fetishization as players invest their belief that ownership of specific cards will bring success in the game and, hence, some degree of validation of their social activities.

\section{Conclusion}

This paper relates to research in progress and it is intended that additional observations and findings will be incorporated into subsequent papers. Games' playing is likely to increase in importance as a leisure activity in Taiwan and elsewhere because of its close relationship with the virtual world. Taiwan, of course, has a highly educated population and a society which has a high degree of acceptance of connectivity. To date, the attempts to locate TCGs within cyberspace have been of only limited success because it has not been able to replicate the pleasurable aspects of face-to-face interactions. However, this does not mean it will necessarily continue along the same path. Games, as part of the mainstream of social activity in Taiwan, seem set to become more fully incorporated into the capitalist system. As this paper has indicated, TCGs have become game objects that are themselves desirable, almost fetishized things for purchase. The cards themselves are also representatives of the circulation of money in society indeed, in many cases, they become items which have a monetary value of their own. They contribute, therefore, to the increasing velocity of capital in circulation. It will be interesting to see how this industry develops and matures in the future. The extent to which young Taiwanese are willing to take their enjoyment in the form of game playing generally and TCGs in particular has become limited as a result of external environmental effects. This is in part a reflection of the withholding of time and money from all forms of cultural productions at a time of poor economic conditions. However, this has disproportionately affected TCGs because of the nature of the business model on which the sector operates. Young people have moved towards lower cost and lower-involvement activities and it will be an interesting study to see how the companies in the industry will respond to this trend and whether they can find means of overcoming this new resistance. 


\section{References}

4K Media, Homepage. (2013). available at: http://www.yugioh.com.

David-Marshall, B., van Dreunen, J. \& Wang, M. (2009). Trading Card Game Industry White Paper: From the $\mathrm{T}$ to the $\mathrm{C}$ to the $\mathrm{G}$ (New York, 2009), available at: http://ebookbrowse.com/trading-cardgame-industry-white-paper-pdf-d23725955.

Davis, N. (2012). Do You Believe in Magic ... the Gathering? The Verge (September 20 th, 2012 ), available at:http://www.theverge.com/2012/9/20/3326708/magic-the-gathering-players-championship.

Loo, J. M. Y., Raylu, N. \& Oei, T. P. S. (2008). Gambling among the Chinese: A Comprehensive Review. Clinical Psychology Review, 28(7), 1152-66.

Martin, B. A. S. (2004). Using the Imagination: Consumer Evoking and The matizing of the Fantastic Imagery. Journal of Consumer Research, 31(1), 136-49.

McArthur, J. A. (2009). Digital Subculture: A Geek Meaning of Style. Journal of Communication Inquiry, 33(1), 58-70.

Raylu, N. \& Oei, T. P. S. (2004). Role of Culture in Gambling and Problem Gambling. Clinical Psychology Review, 23(8), 1087-1114.

Sale, K. \& Zimmerman, E. (2003). Rules of Play: Game Design Fundamentals (Cambridge, MA: The MIT Press).

Walsh, J. \& Techavimol, P. (2009). Following the Korean Model: Management of the Online Computer Games Industry in Thailand. GITAM Journal of Management, 7(3), 1-13.

Winkler, K. W. (2006). The Business and the Culture of Gaming, in Patrick J. Williams, Sean Q. Hendricks and Keith W. Winkler, Gaming as Culture: Essays on Reality, Identity and Experience in Fantasy Games (Jefferson, NC: McFarland and Company, Inc., 2006), 140-53. 